\title{
Ultrasonic Imaging of Pitting using Multilayer Synthetic Aperture Focusing
}

\author{
Martin H. Skjelvareid and Yngve Birkelund \\ Dept. of Physics and Technology \\ University of Troms $\varnothing$ \\ N-9037 Troms $\varnothing$, Norway \\ E-mail: martin.skjelvareid@breivoll.no
}

\author{
Tomas Olofsson \\ Dept. of Engineering Sciences \\ Uppsala University \\ Uppsala, Sweden
}

\author{
Yngvar Larsen \\ Dept. of Earth Observation \\ Northern Research Institute \\ Troms $\varnothing$, Norway
}

\begin{abstract}
Synthetic aperture focusing is known to increase both the lateral resolution and signal-to-noise level of pulse-echo ultrasonic images. In this paper, the use of synthetic aperture imaging for detection and sizing of pitting in a plate is considered. It is assumed that a water layer is present between the transducer and the plate, and that the pitting is on the back side of the plate, seen from the transducer. To account for the different wave velocities of the water and plate layers, one of the recently proposed multilayer synthetic aperture algorithms is used. The algorithm can be adapted to both $2 \mathrm{D}$ (line scan) and 3D (surface scan) data, and these two variations are tested on a number of artificial pittings in an aluminum plate. Comparison of raw and focused images shows that the algorithm significantly improves the lateral resolution, thus enabling an accurate estimation of the pitting shape.
\end{abstract}

\section{INTRODUCTION}

Detection and sizing of corrosion damage is vital when assessing the condition of water pipelines. Since the pipes are usually buried in the ground, they have to be inspected from the inside using an inspection robot. It is often not practically feasible to empty the pipes of water before the inspection, and the inspection method must therefore be compatible with a water-filled pipe. Pulse-echo ultrasound measurements are a natural choice of inspection method, since ultrasound propagates well through water, and since the water layer also acts as a good acoustic couplant for the transducer.

Corrosion damage is often present on both the inside and the outside of the pipe, but in this work the imaging problem is limited to outside corrosion only. To accurately determine the size and depth of a corroded area, high spatial resolution is required, and synthetic aperture focusing techniques (SAFT) provide the means for creating such high-resolution images. These techniques are based on performing several pulse-echo measurements along a line or over a plane, and combining them in post-processing to obtain a focused image.

SAFT techniques were first developed within the field of non-destructive testing during the 1970s and 1980s [1, [2]. The techniques have mainly been used for contact scans, where it can be assumed that the waves propagate through a single, uniform medium. However, in a waterfilled pipeline, the water and the pipe wall constitute two layers of different wave velocity, and this must be taken into account when focusing. A number of different multilayer SAFT algorithms have recently been introduced [3], [4], [5], and in this paper, the algorithm proposed in [3] is evaluated for imaging of outside corrosion in a plate. The algorithm is based on the Phase Shift Migration concept 6], and can be adapted for both the 2D (line scan) case and the 3D (surface scan) case. Both of these are tested here. Since the algorithm assumes that the layers are parallel, a flat plate is used in the experiments, rather than a curved pipe wall. However, we believe that the general results presented here are also relevant in development of SAFT for pipe geometries.

\section{THEORY}

\section{A. Extrapolation of wave fields}

It is assumed that a series of pulse-echo measurements are performed over the plane $z=Z$, and that all reflectors are located in the half-space $z>Z$. At each position $(x, y)$, a pulse-echo measurement is conducted, and the set of measurements are denoted $p(t, x, y, Z)$, where $t$ denotes the two-way travel time of the pulse. The Fourier transform of $p(t, x, y, Z)$ is given by

$$
P\left(\omega, k_{x}, k_{y}, Z\right)=\mathcal{F}_{t, x, y}\{p(t, x, y, Z)\}
$$

where $\mathcal{F}_{t, x, y}$ denotes three-dimensional Fourier transform over the $t, x$ and $y$ axes, $\omega$ denotes temporal frequency, and $k_{x}$ and $k_{y}$ are the wave numbers of the $x$ and $y$ dimensions, respectively. It can be shown [7] that the recorded wave field can be extrapolated to any depth $z=Z+\Delta z$ through the expression

$$
P\left(\omega, k_{x}, k_{y}, Z+\Delta z\right)=P\left(\omega, k_{x}, k_{y}, Z\right) \cdot \exp \left(i k_{z} \Delta z\right)
$$

where $k_{z}$ is given by

$$
k_{z}\left(\omega, k_{x}, k_{y}, c\right)=-\operatorname{sgn}(\omega) \cdot \sqrt{\frac{\omega^{2}}{c / 2}-k_{x}^{2}-k_{y}^{2}} .
$$

In Eq. 3, $c$ denotes the wave velocity of the medium. The wave velocity is divided by 2 to account for the two-way 


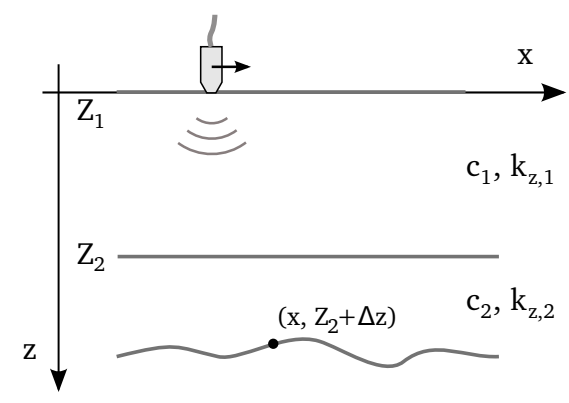

Figure 1. Two-layered geometry.

propagation delay of the pulse-echo measurements. This corresponds to the "exploding reflector" model often used in seismic imaging [7].

\section{B. Imaging}

To obtain a focused image from an extrapolated wave field the imaging condition $t=0$ is applied [7]. Evaluation at $t=0$ corresponds to summation over $\omega$ in the frequency domain, and thus a focused image plane $i(x, y, Z+\Delta z)$ can be obtained from the extrapolated wave field $P\left(\omega, k_{x}, k_{y}, Z+\Delta z\right)$ through the expression

$$
i(x, y, Z+\Delta z)=\mathcal{F}_{x, y}^{-1}\left\{\int_{-\infty}^{\infty} P\left(\omega, k_{x}, k_{y}, Z+\Delta z\right) d \omega\right\}
$$

where $\mathcal{F}_{x, y}^{-1}$ denotes a 2D inverse Fourier transform from the $\left(k_{x}, k_{y}\right)$ to the $(x, y)$ domain.

\section{Imaging in a two-layered geometry}

The extrapolation and imaging procedures described above can be extended to a general multilayered geometry [5], but for a water-filled pipeline, the number of layers is limited to two. Such a two-layered geometry is shown in Fig. 1. where the transducer is scanned over the top of the first layer, at depth $z=Z_{1}$, and the interface between layers 1 and 2 is given by $Z_{2}$. The wave velocities of layer $l$ are denoted by $c_{1}$ and $c_{2}$, and since $k_{z}$ is a function of the wave velocity (Eq. 3), $k_{z, 1}$ and $k_{z, 2}$ is used to denote $k_{z}$ for the two layers. The coordinates of a point in the second layer are given by $\left(x, Z_{2}+\Delta z\right)$, where $\Delta z=z-Z_{2}$. The wave field at the interface $Z_{2}$ is given by

$P\left(\omega, k_{x}, k_{y}, Z_{2}\right)=P\left(\omega, k_{x}, k_{y}, Z_{1}\right) \cdot \exp \left(i k_{z, 1} \cdot\left(Z_{2}-Z_{1}\right)\right)$,

and this can be used for further extrapolation into the second layer,

$$
P\left(\omega, k_{x}, k_{y}, Z_{2}+\Delta z\right)=P\left(\omega, k_{x}, k_{y}, Z_{2}\right) \cdot \exp \left(i k_{z, 2} \Delta z\right) .
$$

The imaging algorithm, which is illustrated in Fig. 2, can be summarized as follows: First the wave field is extrapolated to the interface $Z_{2}$, using Eq. 5 Then, for each depth $Z_{2}+\Delta z$ to be imaged, the wave field is extrapolated downwards with $\Delta z$, using Eq. 6. A focused

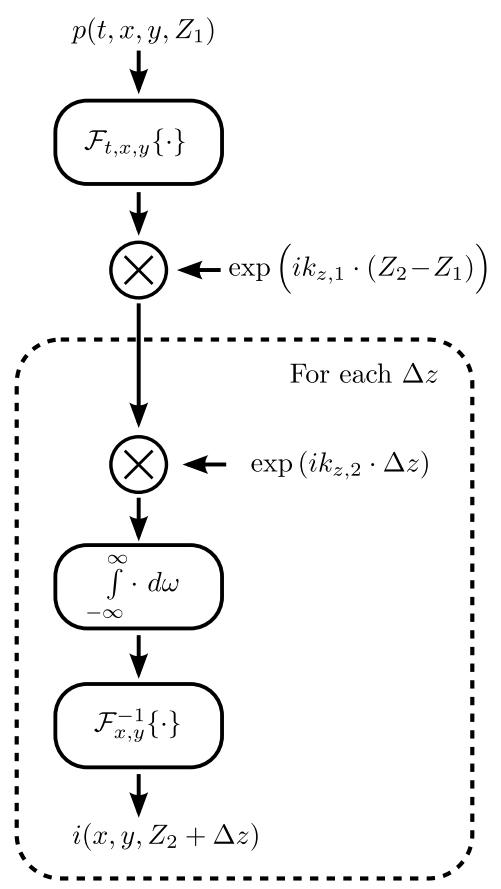

Figure 2. Flowchart for two-layer synthetic aperture focusing algorithm.

image plane $i\left(x, y, Z_{2}+\Delta z\right)$ is obtained by summation over $\omega$ followed by Fourier transformation, as given by Eq. 4 A three-dimensional focused image is created by stacking the focused image planes.

When the transducer is scanned along a line rather than a plane, a $2 \mathrm{D}$ data set $p\left(t, x, Z_{1}\right)$ is obtained, rather than the $3 \mathrm{D}$ data set described in the derivation. The algorithm can be adapted to $2 \mathrm{D}$ data sets by simply omitting the $y$ and $k_{y}$ terms.

\section{EXPERIMENTS}

\section{A. $2 D$ pitting}

Two experiments were conducted, with one designed for the $2 \mathrm{D}$ version of the algorithm, and another for the $3 \mathrm{D}$ version. For the $2 \mathrm{D}$ experiment, two profiles were machined out of a $30 \mathrm{~mm}$ thick aluminum plate. The profiles were made uniform throughout the plate, so that there was thickness variation only along one axis. The profiles are shown in Fig. 3, together with the measurement setup. The plate was immersed in water, and a $\varnothing 6 \mathrm{~mm}, 2.25$ $\mathrm{MHz}$ transducer was scanned in the water layer $50 \mathrm{~mm}$ above the plate surface. A pulse-echo measurement was performed every $0.5 \mathrm{~mm}$.

The leftmost profile was made to have a smooth and rounded surface, while the rightmost profile was made as a number of rectangular steps with sharp edges. These will be referred to as the "round" and "sharp" profile.

The raw data and the corresponding focused image is shown in Fig. 4 Reflections from the profiles are clearly visible, but due the the divergence of the transducer beam, 


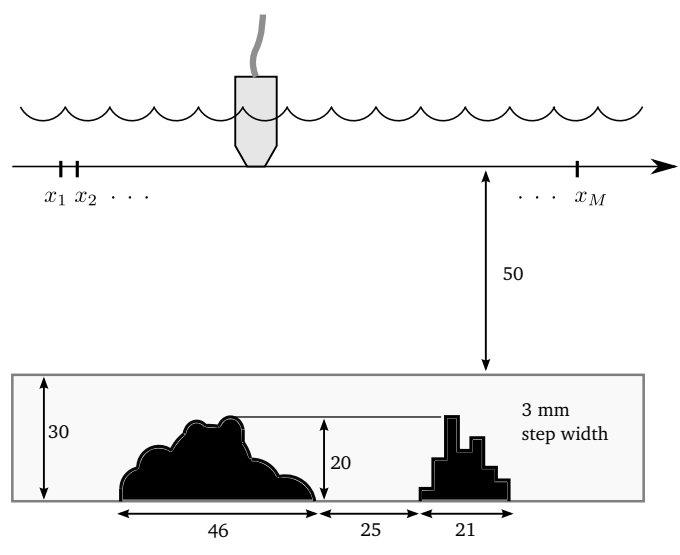

Figure 3. Experimental setup for scan of 2D profiles in aluminum plate. All dimensions are in $\mathrm{mm}$.
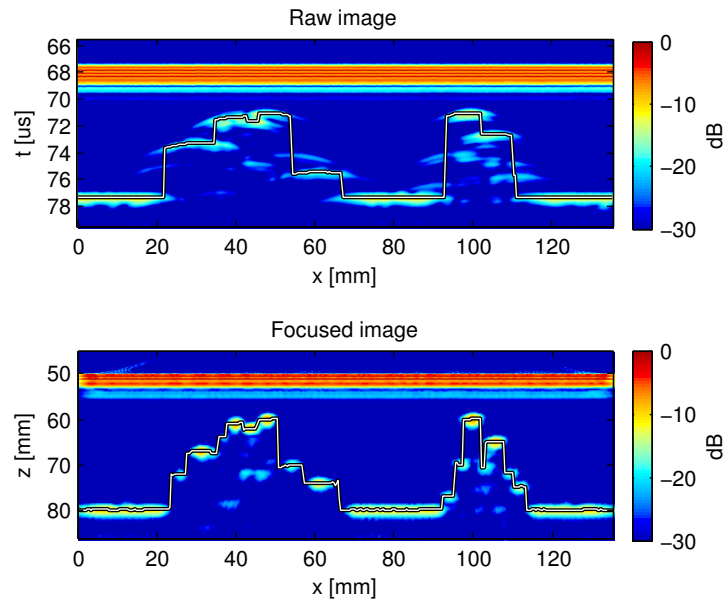

Figure 4. Raw data (top) and focused image (bottom) from scan of $2 \mathrm{D}$ aluminum profiles. The location of maximum amplitude below the front echo is indicated with a black and white line.

they have been "smeared" into a set of curved shapes. This is especially visible for the sharp profile. The focused image is seen to consist of small, separate reflections, rather than a continuous outline of the profiles. These points correspond with sections of the profiles which are relatively flat and parallel to the scanning line. This is to be expected, since surfaces that are tilted relative to the incident angle of the ultrasonic pulse will tend to deflect the pulse, so that little or no echo is reflected back towards the transducer.

To estimate the profile shape from the data, the maximum amplitude below the front surface was detected for each $x$ position. The estimated shapes are indicated with a black and white line. Comparing the lines for the raw and focused images, it can be seen that the focused image yields a much more accurate outline of the profile. However, since only the flat sections of the profiles are imaged properly, the outline of the round profile resembles a number of sharp steps, similar to the sharp profile.

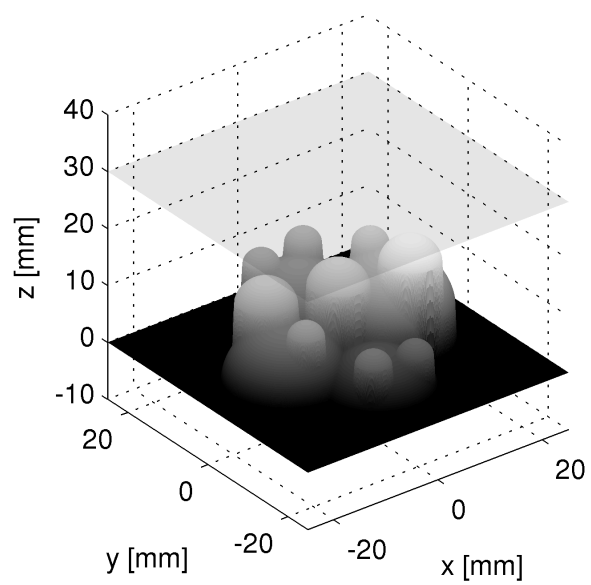

Figure 5. Surface plot of artificial 3D pitting in aluminum plate. Upper (front) surface shown as light gray.

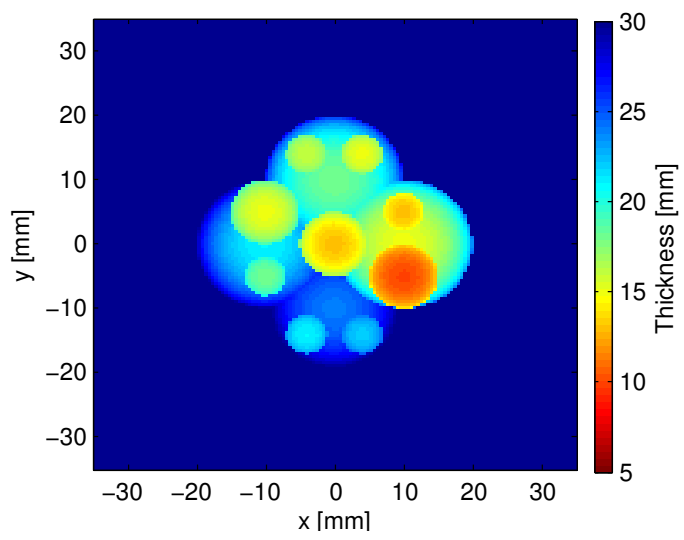

Figure 6. Remaining plate thickness for artificial 3D pitting.

\section{B. 3D pitting}

To test the 3D version of the SAFT algorithm, several pits of various sizes were machined out in a $30 \mathrm{~mm}$ aluminum plate. The resulting simulated pitting profile is shown as a $3 \mathrm{D}$ surface plot in Fig. III-B and as a plot of remaining plate thickness in Fig. IIII-B. The test block was immersed in water and scanned under the same experimental conditions as for the $2 \mathrm{D}$ profile, except that it was scanned in both the $x$ and $y$ directions. The spatial sampling interval was $0.5 \mathrm{~mm}$ along both axes.

The raw data was processed with the SAFT algorithm, and for both the raw and the focused data sets the maximum amplitude below the front surface was detected. The plate thickness was estimated as the difference in depth between the front surface echo and the maximum amplitude reflection. Figures 7(a) and 7(b) show plots of the maximum amplitude below the front surface, while Figs. $7(\mathrm{c})$ and $7(\mathrm{~d})$ show the corresponding estimated thickness of the plate.

Studying the raw data amplitude image of Fig. 7(a), it 
can be seen that the back-scattered amplitude is generally lower in the pitting area, due to increased scattering. The general shape of the area can be seen, but it is hard to identify any details. In the focused amplitude image in Fig. 7(b) there are localized areas with strong backscattering, which closely match the local peaks of the pitting profile. Note that in areas where the pitting surface is tilted relative to the front surface, for example along the circumference of the pitting, the amplitude is very low.

The images of the estimated thickness should ideally be identical to Fig. III-B. However, the thickness estimated from raw data shown in Fig. $7(\mathrm{c})$ is a collection of oddly shaped patches, hardly similar to Fig. III-B Compared to this, the focused thickness plot shown in $7(\mathrm{~d})$ is relatively accurate, although the individual peaks appear somewhat flattened. Sections where the back-scattered amplitude was below $-20 \mathrm{~dB}$ were masked out with a white color, since they were found to yield erroneous thickness estimates.

The lateral resolution of the focused images is generally seen to be slightly better in the $x$ direction than in the $y$ direction. It is assumed that this is due to a difference in the scanner positioning accuracy between the two axes.

\section{Conclusions}

It has been shown that the use of multilayer synthetic aperture techniques can significantly increase the lateral resolution in pulse-echo images of back surface pitting in a plate. The focusing operation makes it possible to accurately determine the size and depth of the individual pittings, and the results are seen as promising with regard to possible imaging of corrosion in pipelines.

\section{REFERENCES}

[1] S. Doctor, T. Hall, and L. Reid, "SAFT - the evolution of a signal processing technology for ultrasonic testing," NDT International, vol. 19, no. 3, pp. 163-167, June 1986.

[2] K. Langenberg, M. Berger, T. Kreutter, K. Mayer, and V. Schmitz, "Synthetic aperture focusing technique signal processing," NDT International, vol. 19, no. 3, pp. 177-189, June 1986.

[3] T. Olofsson, "Phase shift migration for imaging layered objects and objects immersed in water," IEEE Transactions on Ultrasonics, Ferroelectrics, and Frequency Control, vol. 57, no. 11, pp. 2522-2530, November 2010.

[4] M. H. Skjelvareid and Y. Birkelund, "Ultrasound imaging using multilayer synthetic aperture focusing," in ASME 2010 Pressure Vessels and Piping Conference, vol. 5, 2010, pp. 379-387. [Online]. Available: http://dx.doi.org/10.1115/PVP2010-25338

[5] M. H. Skjelvareid, T. Olofsson, Y. Birkelund, and Y. Larsen, "Synthetic aperture focusing of ultrasonic data from multilayered media using an omega-k algorithm," IEEE Transactions on Ultrasonics, Ferroelectrics, and Frequency Control, vol. 58, no. 5, pp. 1037-1048, 2011.

[6] J. Gazdag, "Wave equation migration with the phase-shift method," Geophysics, vol. 43, no. 7, pp. 1342-1351, 1978

[7] J. F. Claerbout, Imaging the Earth's Interior. Blackwell Scientific Publications, 1985.

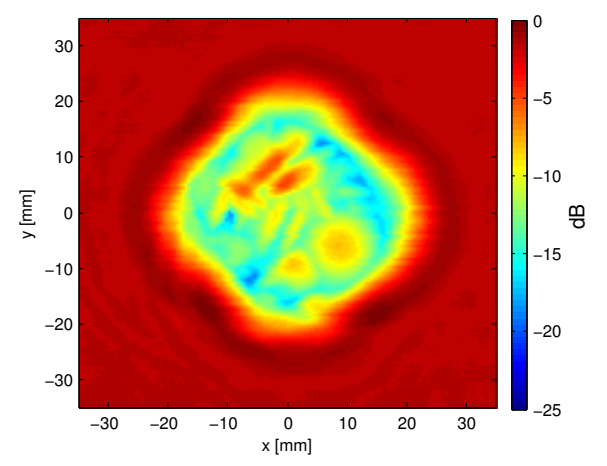

(a) Raw data, max. amplitude below front echo

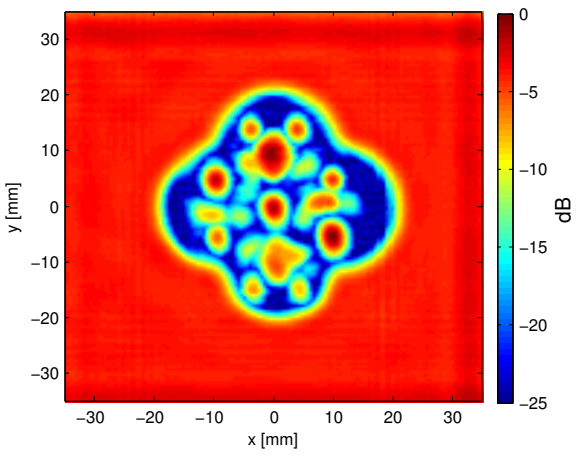

(b) Focused image, max. amplitude below front echo

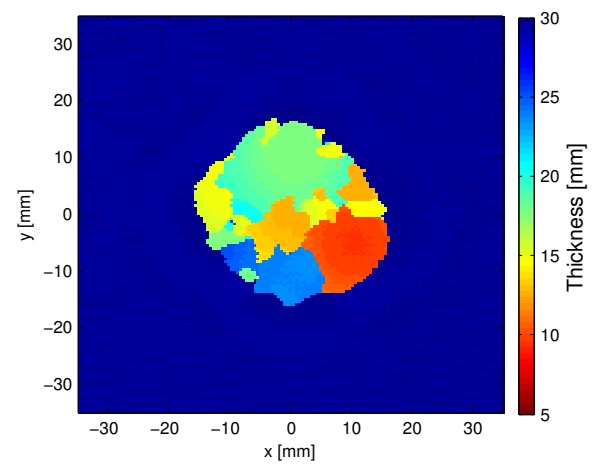

(c) Raw data, estimated thickness

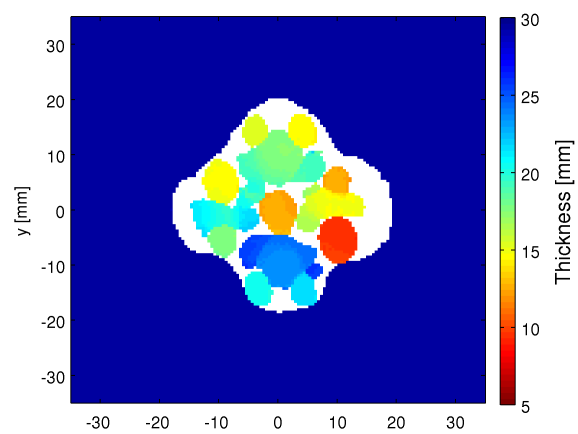

(d) Focused image, estimated thickness. Reflections below $-20 \mathrm{~dB}$ masked out with white color

Figure 7. Amplitude and thickness plots based on the maximum amplitude detected below the surface echo of the aluminum plate. 